\title{
Umbruchperiode - Krisentyp - Staatsmonopolistisch deformierte Vergesellschaftung
}

\section{Von HEINZ JUNG (Frankfurt am Main)}

In der gegenwärtigen Diskussion über die zukünftige Entwicklung der BRD kommt ihrer Charakterisierung als krisenhafte Umbruchperiode besondere Bedeutung zu. ${ }^{1}$ Hierzu sollen einige zentrale Argumente vorgestellt werden, die sich als Konsequenz aus der Veränderung des Systems der Produktivkräfte ergeben und die dann auf den Krisentyp des heutigen Kapitalismus zu beziehen sind. Zentrale Konfliktfelder ergeben sich dabei aus der Spezifik staatsmonopolistisch deformierter Vergesellschaftung. Schlieflich ist es entscheidend, welche möglichen Konstellationen sozialer und politischer Kräfte der Veränderung sich unter diesen Bedingungen abzeichnen. Diesen Fragen ist der nachfolgende Beitrag gewidmet.

\section{Umbruchperiode?}

Die moderne Militärtechnologie und die neuen Waffensysteme haben unter den gegebenen politischen und sozialen Bedingungen das Überleben der Menschheit und die Erhaltung des Friedens zum wichtigsten und aktuellsten Problem werden lassen. In dieser Zwecksetzung sind die Produktivkräfte in Destruktivkräfte umgeschlagen. Dies bedeutet in der Tat den radikalsten Umbruch in den Existenzbedingungen nicht nur einer Klasse oder eines Landes, sondern der gesamten Menschheit. Diese Entwicklungen verweisen auf den gewaltigen Schub von Wissenschaft und Technik, der in den letzten 40, 50 Jahren stattgefunden und sich am schnellsten und intensivsten im Militär- und Rüstungssektor realisiert hat. Nach den bekannten gewaltigen Rüstungsprogrammen des Pentagon muf in den kommenden Jahren mit einer weiteren Beschleunigung dieses Schubs gerechnet werden. Im zivilen Sektor und damit in der Breite der Volkswirtschaft ist die Durchsetzung der wissenschaftlich-technischen Revolution erst punktuell und in den Anfängen. Aber unter dem Druck der internationalen Konkurrenz ist in den internationalen Konzernen und den entwickelten kapitalistischen Ländern ein sich kumulierender Prozeł in Gang gekommen, der $z \mathfrak{u}$ einschneidenden Änderungen im System der Produktivkräfte und der gesellschaftlichen Arbeit führen wird.

Die Automation und Roboterisierung wird sich vorerst in den Kernbereichen der industriellen Massenproduktion durchsetzen und zur Verdrängung lebendiger Arbeit aus der Produktionslinie und dann - gleich- oder fortlaufend - aus den vor- und nachgelagerten Bereichen führen. Das Revolutionäre der Änderungen im Produktions- und Arbeitsprozef 3 mit der Verbreitung der Mikroprozessoren- und Optotechniken besteht darin, dał die Steuerungs-, Überwachungs-, Gedächtnis-, Informationsverarbeitungs- usw. -funktionen, also traditionell geistig-psychische Funktionen der

I Vgl.: Marxistische Studien. Jahrbuch (im folgenden Jb) des IMSF 9. Frankfurt a. M. 1985 (Siehe auch die Rezension zu diesem Jahrbuch in diesem Heft. Der vorliegende Beitrag von Heinz Jung, dem Direktor des Instituts für Marxistische Studien und Forschungen (IMSF) in Frankfurt a. M., stützt sich auf seinen Einleitungsartikel im Jahrbuch 9 (1985): Umbruchperiode? Die BRD bis zum Jahr 2000 - Die Red.) 
Arbeitskraft, auf stoffliche Systeme übertragen und vernetzt werden können und damit eine gewaltige Potenzierung ihres Wirkungsgrades hervorrufen.

Dies wird die Berufsstrukturen, die Qualifikationen und die Typen der Arbeitsverausgabung betreffen. Die Unterschiede zwischen früher gegeneinander abgeschotteten Betriebsbereichen (Werkstatt, Büro, technische Entwicklung usw.) werden eingeebnet werden. Die neuen Technologien ermöglichen eine größere Variationsbreite in der Gestaltung der Arbeitsorganisation und des Produktionsablaufes. Der technologische "Sachzwang" kommt damit unter einen stärkeren Legitimationsdruck, was in der Tendenz schon heute überall dort zu erkennen ist, wo die neuen Systeme eingeführt werden.

Die Rolle der Wissenschaft als einer mittelbaren und unmittelbaren Produktivkraft wird weiter zunehmen. Vom wissenschaftlichen Fortschritt wird zunehmend die Umgestaltung der Produktion und der Produkte beeinfluft werden. Die Wissenschaft wird sich weiter ausdifferenzieren und neue Bereiche durchdringen. Randbereiche der Forschung können mit ihren weitreichenden Anwendungsmöglichkeiten und Ergebnissen schnell ins Zentrum des Interesses und der Auseinandersetzung rükken (z. B. Genbiologie). Die Frage gesellschaftlicher Kontrolle der Wissenschaft erlangt damit wachsende Brisanz und Bedeutung. Dies gilt nicht nur für die Naturund Technikwissenschaften, sondern auch für die Gesellschaftswissenschaften. Dies wird schon heute bei den neuen Informations- und Kommunikationssystemen deutlich, deren Anwendungsbedingung die wissenschaftlich-analytische Durchdringung technologischer, ökonomischer und sozialer Zusammenhänge ist.

Während in der unmittelbaren Produktion ein geringer werdender Teil der gesellschaftlichen Arbeit angewendet werden wird, und zwar sowohl im betrieblichen als auch im volkswirtschaftlichen Rahmen, wird der Umfang der vor- und nachgelagerten Funktionen des produktiven Gesamtarbeiters wachsen. Unter den Bedingungen zunehmender Widersprüche des kapitalistischen Wirtschaftsprozesses ist es unvermeidlich, da\}̧ auch die kommerzielle Sphäre weiter wachsen wird, obwohl ähnlich wie in der Produktion auch die Bereiche der Realisierung der Waren zunehmender Rationalisierung zugänglich und unterworfen werden. Eine Haupttendenz der Umschichtung der gesellschaftlichen Arbeit wird die Ausweitung der reproduktiven Sphäre betreffen, also jener Bereiche, die sich auf die Reproduktion der Arbeitskraft in einem weiteren Sinne beziehen. Hierin drückt sich eine Entwicklungsbedingung der modernen Produktivkräfte aus, die in der Tendenz die allseitige Entwicklung der Individualităt und Disponibilität der Träger der lebendigen Arbeitskraft erforderlich macht.

Diese Prozesse sind keinesfalls neu, sondern schon seit längerer Zeit erkennbar. Sie dürfen in ihrer kurzfristigen gesamtgesellschaftlichen Wirkung auch keinesfalls überzeichnet werden, aber dennoch gehen von ihnen die strukturbestimmenden Wirkungen aus. Ihre Durchsetzung unter staatsmonopolistischen Bedingungen im allgemeinen und unter verschärften kapitalistischen Krisenbedingungen im besonderen kann nur um den Preis zunehmender sozialer Spannungen und Friktionen erfolgen. Um sie werden sich wesentliche Konfliktfelder der kommenden Periode aufbauen. Sie betreffen die Fragen der Arbeitslosigkeit, der Qualifikationsentwertung auf der einen und der neuen Qualifikationsanforderungen auf der anderen Seite, den sozialpolitisch zu vermittelnden Ausbau der Reproduktionssphäre, was eine Ausweitung der Sozialquoten notwendig macht und auf die Verwertungsinteressen des Kapitals stößt usw. Für die Herausbildung des neuen Produzententyps wird die Reproduktionskomponente ein gröferes Gewicht als jemals zuvor in der Vergangenheit haben.

Schon heute ist erkennbar, daf̧ neue Züge in der Lebensweise und in den Lebensorientierungen der Lohnabhängigen auftreten. Sie setzen sich jedoch nicht gleichmäfjig in allen Schichten durch, sondern äufern sich in einer weiteren Ausdifferenzierung des Lebensmilieus der verschiedenen Schichten, Generationsgruppen usw. Der methodische Ausgangspunkt für die Erfassung dieser Prozesse sind die Veränderungen in der Sphäre der gesellschaftlichen Produktion, von ihnen geht auch die Dynamik der 
Umgestaltung der Bedürfnisse aus. Es gilt der Zusamménhang: neue Arbeitsweise neue Reproduktionsweise - neue Lebensweise. Aber dieser Zusammenhang darf nicht schematisiert werden, da er gegenüber dem Individuum und in seiner Sozialisation in umgekehrter Reihenfolge wirksam wird. Die Rolle des Bildungssystems wächst. Mit der Ausweitung der Freizeit erlangen soziale Bindungen außerhalb der Arbeitsbeziehungen einen größeren Raum und größeres Gewicht.

Wenn von neuer Lebensweise und der Rolle, die die neuen Technik-, Informationsund Kommunikationssysteme dabei spielen, die Rede ist, dann muf beachtet werden, dał die gesellschaftlichen Widersprüche hier in spezifischer Weise zum Ausdruck kommen: Der Tendenz zur Ausprägung der Individualität steht die Ausbeutung und .Herrschaft im Betrieb gegenüber, dem Drang zur Selbsttätigkeit die Kommerzialisierung der Freizeit durch das Kapital, die kulturelle Entfaltung wird in das Konzept der durch Massenmedien und Reklame gestanzten Stereotypen geprefjt usw. In diesen Widersprüchen werden sich die Bedürfnisse der kommenden Periode entwickeln und in vieler Hinsicht das kulturelle Milieu der Gesellschaft formen.

\section{Der Krisentyp des heutigen Kapitalismus}

Die kapitalistischen Wirtschaftskrisen drücken aus, da\} die kapitalistischen Produktionsverhältnisse nur um den Preis gesellschaftlicher Verluste zur Anwendung und Weiterentwicklung der Produktivkräfte in der Lage sind. Der Preis ist die Brachlegung, Entwertung, Vernichtung vorhandener gegenständlicher und lebendiger Produktionspotentiale. Nur auf diesem Wege können ökonomische Disproportionen, die das Ergebnis der kapitalistischen Widersprüche sind und einer profitablen Kapitalverwertung im Wege stehen, zeitweilig bereinigt und damit die Bedingungen weiterer Kapitalverwertung wiederhergestellt werden.

Wenn diese Reinigungsfunktion durch die zyklische Krise nicht erfüllt wird, ist dies ein Indiz für tieferliegende strukturelle Probleme und für übergreifende Defekte im ökonomischen Regulierungsmechanismus. Das ist in der gegenwärtigen Periòde offenkundig der Fall. Die Krise kann deshalb als Struktur- und Regulierungskrise des staatsmonopolistischen Wirtschaftssystems angesehen werden, die in der krisenhaften Entwicklung der internationalen Wirtschaftsbeziehungen kulminiert. ${ }^{2}$

Will man diesen Prozefy verstehen, dann muf die Struktur der Produktionsverhältnisse auf dem heutigen staatsmonopolistischen Kapitalismus (im folgenden: SMK) beachtet werden. So ist die Herrschaft der Monopole zwar keine neue Erscheinung, aber der starke Schub der internationalen Monopolisierung fällt in die 60er und 70er Jahre. Die an den Monopolinteressen ausgerichteten staatsmonopolistischen Wirtschaftsaktivitäten orientieren sich unter diesen Bedingungen zunehmend an der Aufenexpansion des Monopol- und Finanzkapitals und der internationalen Konkurrenzfähigkeit. Es entsteht die Situation, in der es zur "Abkoppelung" der Monopole vom nationalen Reproduktionsprozef kommen kann und auf binnenwirtschaftliches Wachstum und Stimulierung der Investitionstätigkeit gerichtete staatliche Wirtschaftsaktivität nicht greift.

2 Vgl. zur neueren marxistischen Krisenanalyse und Diskussion: Grofe Krisen des Kapitalismus - Lange Wellen der Konjunktur? (Beiträge von U. Dolata, J. Goldberg, D. Klein, J. Priewe, W. Schwarz, H. Wagner). In: IMSF-Informationsberichte 41. S. 26. Frankfurt a. M. 1985; S. Menschikow: Die Strukturkrise der Wirtschaft des Kapitalismus (russ.). In: Kommunist (Moskau). Nr. 4/1984; J. Huffschmid: Die Wirtschaftskrise in der BRD: Tendenzen und Perspektiven. In: IMSF-Jb 6 (1983). S. 235 ff.; A. Sörgel: Regulierung und Regulierungskrise der kapitalistischen Wirtschaft im Kontext der Marxschen politischen Ökonomie. In: IMSF-Jb. Sonderband I (1983). S. 110 ff.; J. Höhme: Probleme des gegenwärtigen kapitalistischen Krisenzyklus. IPW-Forschungshefte (Berlin). Nr. 2/1982; ferner sei verwiesen auf die jährlich erscheinenden Analysen in den Memoranden der Arbeitsgruppe Alternative Wirtschaftspolitik. 
Dies fällt mit der zunehmenden, wenn auch äuferst widersprüchlichen Durchsetzung des wissenschaftlich-technischen Fortschritts in der Produktion und Wirtschaft zusammen, also dem Typ intensiver Reproduktion bei Intensivierung und Effektivierung der Produktion. Darin ist die Veränderung der Proportionen der Kapitalbestandteile eingeschlossen, die Freisetzung von Kapital und die Überakkumulation von Geldkapital, das nach Verwertung drängt. Unter diesen Bedingungen muf sich das Marktproblem zuspitzen, wenn eine funktionierende Kapitalwanderung nicht die Umleitung der freigesetzten Kapitale in profitable Anlagefelder, damit die Schaffung neuer Arbeitsplätze, Lohneinkommen usw. gewährleisten kann. Gerade das ist aber nicht der Fall. Die Investitionstätigkeit konzentriert sich auf die Rationalisierung; die Verfahrensinnovation vernichtet bedeutend mehr Arbeitsplätze als sie neue schafft; sie bewirkt jedoch auch keine breite Ankurbelung der Wirtschaft von seiten der Produktionsmittelproduktion, weil die neue Technik durch einen relativ schmalen Sektor getragen wird und gerade dort die Rationalisierung bedeutende Fortschritte gemacht hat. ${ }^{3}$ Das Wachstum bleibt infolge der Nachfrageschwäche niedrig, die Produktivität wächst, die Arbeitslosigkeit bleibt hoch und nimmt weiter zu. Das ist selbst die Situation in zyklischen Aufschwungperioden. Auf diesen Krisentyp hat der SMK der BRD mit der sogenannten Modernisierungsstrategie unter Weltmarktdominanz reagiert, was die Präferenz der Wirtschaftspolitik für die Förderung der Profitproduktion (Angebotspolitik) bei gleichzeitiger gesamtwirtschaftlicher Priorität für im internationalen Vergleich niedrige Lohn- und Preissteigerungsraten einschlief3t.

Ein Grundproblem der derzeitigen Krise ist die strukturelle Überakkumulation von fixem Kapital, die in Überkapazitäten, sektoralen Krisen usw. zum Ausdruck kommt. Es kommt hier jedoch für die Einzelkapitale nicht zu einer umfassenden Kapitalentwertung, da diese meist durch Monopole beherrschten Branchen mittels monopolistischer Preispolitik, Kartellierung usw. sowie mittels der Mobilisierung des Staats den Entwertungsdruck von sich abwehren können. Der Prozeł der Überakkumulation und die beherrschende Rolle des Finanzkapitals war seinerzeit für Lenin, aber auch andere Kritiker des Imperialismus', der wesentlichste ökonomische Grund, von der Zunahme parasitärer Züge im Imperialismus zu sprechen. Dieser Parasitismus wird in längeren Prosperitätsphasen verdeckt, tritt jedoch wieder verstärkt und relativ unverhüllt in Krisenphasen wie der derzeitigen in Erscheinung.

Andererseits stehen vor der Gesellschaft Aufgaben, die die Mobilisierung gewaltiger Ressourcen erforderlich machen. Dies betrifft die materiellen Voraussetzungen des Produktionsprozesses und des Lebensprozesses der Gesellschaft im umfassenden Sinne sowie die Umstellung der Produktion unter ökologischen und ressourcensparenden Gesichtspunkten. Hier sind jedoch vom Standpunkt des Gesamtkapitals und der Monopole im grofen und ganzen vorwiegend nur infrastrukturelle Małnahmen akzeptabel. Als Fazit bleibt, da\} unter den heutigen Bedingungen der Kapitalwanderungsmechanismus (Preis- und Kapitalanlagenkonkurrenz) nicht mehr im Sinne einer Ressourcenallokation zur optimalen Entwicklung und Nutzung der nationalen Ressourcen zu funktionieren vermag und daf auch der staatsmonopolitische Regulierungsmechanismus an die harten Grenzen der Interessen des Monopol- und Finanzkapitals stöft, was die Regulierungskrise forciert.

Bleibt vor allem die Verlagerung der Widersprüche auf das äußere Feld. Dies ist der Weg, den das Monopolkapital der BRD bisher nicht erfolglos beschritten hat. Der Drang nach dem Weltmarkt, in ausländische Kapitalanlagen und Schuldentitel ist auch der Weg, den das überakkumulierte Geldkapital beschreitet. Was die Folgen

3 Zur Entwicklung dieser Prozesse unter Branchengesichtspunkten vgl.: Beiträge des IMSF 7 : Umbruch im Produktionsbereich? Branchenanalysen (Red.: J. Goldberg : Beitrãge von D. Düe, F. Fiehler, J. Goldberg, G. Hautsch, P. Herrmann, A. Leisewitz, N. Müller, L. Selg). Frankfurt a. M. 1985

4 Vgl. W. I. Lenin: Der Imperialismus als höchstes Stadium des Kapitalismus. In: Werke. Bd. 22. S. 199 ff.; J. A. Hobson: Der Imperialismus. (London 1902) Köln 1968 
für die "dritte Welt" betrifft, so sind sie hinlänglich bekannt: Verschuldung gigantischen Ausmafes, Transfers in einem Ausmał, die den Nettokapitalzufluf̧ übersteigen, Schuldenmoratorien mit IWF-Auflagen, die die Wirtschaft lähmen und die Abwärtsspirale beschleunigen. Die Weltmarktflaute und die Krise auf den Absatzmärkten der kapitalistischen Länder drückt wiederum die Preise für die Exportwaren der Länder der "dritten Welt" und verstärkt dort wiederum den Ausbeutungsdruck, die Tendenz zur Monokultur und zum Raubbau an den nationalen Ressourcen. Mit dem kapitalistischen Entwicklungsweg und der ökonomischen Einbindung in das System des Imperialismus kann die Masse dieser Länder offensichtlich die Unterentwicklung der Produktivkräfte als Erbe des Kolonialismus und imperialistischer Unterdrückung nicht überwinden. Das setzt jedoch auch der Entwicklung des Weltmarktes restriktive Grenzen.

Auf der anderen Seite hat sich die Konkurrenz zwischen den imperialistischen Ländern und Zentren verschärft. Bekanntlich waren die USA im vergangenen Jahrzehnt zum Magneten für finanzkapitalistische Anlagen der relativ überschüssigen Geldkapitale der imperialistischen Länder und der erdölexportierenden Entwicklungsländer geworden. Der Motor dieses Prozesses, der allerdings nur infolge der Rolle des US-Dollar im internationalen Währungssystem möglich war, war vor allem das Aufrüstungsprogramm der US-Regierung und dessen Kreditfinanzierung.

Fragt man die derzeitigen Krisenprozesse auf ihre Zukunftstendenzen ab, dann ist ihre kurz- und mittelfristige Bereinigung nicht in Sicht. Selbst wenn man den relativ optimistischen Fall einer nur geringen Verschärfung des Zyklus annimmt, in dessen Rhythmus sich die Krisenprozesse nach wie vor bewegen werden, steht eine Zunahme der ökonomischen und sozialen Probleme für die BRD ins Haus.

\section{Staatsmonopolistisch deformierte Vergesellschaftung}

Vergesellschaftung aus der Sicht des Individuums bedeutet, daf seine Existenz- und Lebensbedingungen, sein ganzes Leben zunehmend durch gesellschaftliche Zusammenhänge und Prozesse konstituiert werden. Dies ist die historische Tendenz der gesellschaftlichen Entwicklung, die sich mit dem Kapitalismus beschleunigt. Die Grundlage dieses Prozesses ist die Vergesellschaftung der Gewinnung des materiellen Lebens, des Stoffwechsels mit der Natur, der Produktion. Je mehr sich der materielle und geistige Reichtum der Gesellschaft entwickelt und sich die materiellen, sozialen und geistigen Bedürfnisse entfalten, entstehen die objektiven Voraussetzungen für die Herausbildung allseitiger menschlicher Individualität für alle Mitglieder der Gesellschaft und nicht mehr nur für privilegierte gesellschaftliche Gruppen.

Solange und soweit das individuelle Privateigentum an Produktionsmitteln, die durch die eigene Arbeit der Eigentümer in Bewegung gesetzt wurden, die Form der materiellen Produktion war, konnte der Produktions- und Arbeitsprozef - abgesehen von den wechselnden Naturbedingungen - unter der Kontrolle der Produzenten bleiben. Die gesellschaftlichen Zwänge machten sich, ähnlich den Naturgewalten, von "aufen" geltend. Soweit sich schon die Waren- und Geldwirtschaft durchgesetzt hat, wird der gesellschaftliche Zusammenhang privater Produzenten über den Markt wirksam, der gegenüber dem Privateigentümer von vornherein als fremde, unkontrollierbare Macht auftritt.

Mit der kapitalistischen Produktionsweise gestaltet das Kapital durch Maschinerie und Fabrik den Arbeitsprozef um, verwandelt ihn in einen arbeitsteilig gegliederten, hierarchisch strukturierten Mechanismus von Detailarbeiten, verleiht der Produktion damit gesellschaftlichen Charakter und unterwirft die unmittelbaren Produzenten als Lohnarbeiter seinem Kommando und der Ausbeutung. Es ist unter diesen Bedingungen unvermeidlich, daf die vergesellschafteten Zusammenhänge der Produktion gegenüber dem Produzenten als ihm entfremdete Mächte in Erscheinung treten, die nicht mehr seiner Kontrolle unterliegen und ihn in das Korsett ausgebeuteter und beherrsch- 
ter Lohnarbeit zwängen. Die freie Arbeit des Kleineigentümers ist durch die unterdrückte Lohnarbeit abgelöst. In diesem Kontrast und in dieser gesellschaftlichen Form erscheint vergesellschaftete Arbeit per se als entfremdete und unfreie Arbeit.

Deshalb denunziert alle an der individuellen Arbeit des Kleineigentümers orientierte Gesellschaftskritik von Rousseau bis zur Gegenwart mit ihrer knechtenden sozialen Form den gesellschaftlichen Charakter überhaupt. Diese Kritik muf immer rückwärtsgewandt und im negativen Sinne utopisch bleiben, weil sie, mit welchen Begründungen auch immer, die Fortschritts- und Emanzipationspotenzen der Vergesellschaftung leugnet und auf die Auflösung der Zusammenhänge vergesellschafteter Produktion zielt. 5 Demgegenüber steht im Zentrum marxistisch-leninistischer Kritik der knechtende, die individuelle Entfaltung unterdrückende Charakter der kapitalistischen Form der Vergesellschaftung. Die Befreiung der Arbeit und des Produzenten ist in dieser Sicht nicht die Rückkehr zur individuellen oder Kleingruppenproduktion, sondern die Aufhebung der Lohnarbeit und des kapitalistischen Eigentums und damit auch die Befreiung gesellschaftlicher Arbeit von den Fesseln des Kapitals. Dies ist eine Kritik nach vorn, die Perspektive der sozialistischen und kommunistischen Neuorganisation der Gesellschaft auf der Grundlage der Entwicklung gesellschaftlicher Produktion und ihrer Unterwerfung unter die bewufte Kontrolle der Gesellschaft.

Es liegt in der inneren Natur der kapitalistischen Produktionsweise, daf sie mit der kapitalistischen Produktion den gesellschaftlichen Charakter der von ihr entwickelten und angewandten oder auch blockierten Produktivkräfte mit dem oder gegen den Willen der in ihr herrschenden Klasse auf immer höhere Stufen heben muf und dabei Formen der Anpassung der sozialökonomischen Strukturen und ihrer sozialen und politischen Organisation hervortreibt. Es sind dies vom Standpunkt gesellschaftlichen Fortschritts deformierte Formen der Vergesellschaftung, weil sie durch die Widersprüche und den Ausbeutungs- und Herrschaftscharakter der kapitalistischen Aneignungsweise geprägt sind. Dies gilt auch und gerade für den SMK als gegenwärtige Entwicklungsphase der kapitalistischen Gesellschaft. ${ }^{6}$ Deshalb ist die Kritik des heutigen Kapitalismus auch die Kritik an den staatsmonopolistisch deformierten Formen der Vergesellschaftung.

Diese Kritik gewinnt vor allem in der sich abzeichnenden Umbruchphase Aktualität, weil hier vom Standpunkt des SMK der Umbruch in den Vergesellschaftungsformen auf die Tagesordnung tritt. Soweit die Vergesellschaftung im Rahmen des bürgerlichen Repräsentativsystems durch den Staat organisiert wird, können dabei immer in mehr oder weniger grofem Małe und abhängig von den politischen und sozialen Kräfteverhältnissen Interessen der arbeitenden Klasse wirksam werden. Dies gilt für die sozialpolitischen Apparate im weiteren Sinne, aber nicht nur für diese. Verstaatlichung ist eine Form von Vergesellschaftung, die sich der Kontrolle der Einzelmonopole entzieht. Sie ist damit aber keine Vergesellschaftung jenseits des Kapitalismus, da nun die Kontrolle durch die Repräsentanz des Gesamtkapitals ausgeübt wird. Demgegenüber bleiben die durch die Monopole und das Privatkapital organisierten Vergesellschaftungsformen unter der unmittelbaren Kontrolle des Privatkapitals und der Profitinteressen. Es handelt sich also um Vergesellschaftungsformen im Rahmen des staatsmonopolistischen Systems, die gleichwohl Gegenstand der Klassenauseinandersetzungen sind und nicht nur "innerkapitalistische" Angelegenheiten. Dies kann verdeutlicht werden an der Auseinandersetzung um die Organisationsformen der elektronischen Massenmedien. Wiewohl also z. B. der öffentlich-rechtliche Status von

5 Vg1. zu den ideologischen Grundmustern: R. Steigerwald: Protestbewegung. Streitfragen und Gemeinsamkeiten. Frankfurt a. M. 1982

6 Vgl. H. Jung: Deformierte Vergesellschaftung. Zur Soziologie des staatsmonopolistischen Kapitalismus. Berlin/Frankfurt a. M. 1985; H. Jung: "Korporatismus" statt "Etatismus"? Staatsmonopolistische Vergesellschaftung und politischer Utberbau heute. In: IMSF-Jb 4 (1981). S. $11 \mathrm{ff}$. 
Rundfunk und Fernsehen eine Form staatsmonopolistischer Vergesellschaftung bleibt, ist er in anderer Art demokrätischen Interessen zugänglich, als dies bei einer privatmonopolistischen Struktur der Fall sein könnte.

Es kann kaum ein Zweifel daran bestehen, daß mit der neuen Technik, mit der unter gegebenen Bedingungen ambivalenten Entfaltung der Potenzen des wissenschaftlich-technischen Fortschritts die Durchsetzung sozialer Einsicht, Vorsicht und Kontrolle in stärkerem Małe in das Zentrum der Auseinandersetzungen rücken muf - und damit auch die Vergesellschaftungsformen. Das gilt auch für die Formen sozialpolitischer Aktivitäten bzw. die gesellschaftlichen Formen der Reproduktion der Arbeitskraft und der Bevölkerung schlechthin. Unter Bedingungen der Krise und der Restriktion des Leistungsniveaus tritt auch bei den staatlichen sozialpolitischen Apparaten die repressive Seite gegenüber den Individuen und sozialen Gruppen stärker hervor. Der Kampf gegen den Repressionscharakter schliefyt es nicht aus, daf vom Standpunkt demokratischer Interessen jene Formen Präferenz erhalten können (Kommunalisierung anstatt zentralstaatlicher Apparate, gesellschaftliche Organisationen anstatt Staatsregie usw.), die in stärkerem Małe der direkten Kontrolle und Demokratie zugänglich sind. Zentralisierung und Groforganisation können dabei ebensowenig fetischisiert werden wie Dezentralisierung und Kleingruppen. Die Vergesellschaftung kann sich in unterschiedlichen Formen realisieren, und der demokratische Charakter ist nicht an klein oder grof gebunden.

In einer kapitalistischen Gesellschaft werden sich die "Sachzwänge" der Profitlogik, sei es über die Konkurrenz und den Markt, sei es innerbetrieblich über die Umgestaltung der Arbeits- und Produktionsprozesse, immer wieder mit elementarer Gewalt Geltung verschaffen. Je mehr sie aber zur Durchsetzung Formen staatsmonopolistischer Vergesellschaftung bedürfen, desto mehr werden sie der sozialen und politischen Kritik zugänglich. Dies schafft generell eine neue Situation, weil sich nun die sozialistische Kritik nicht mehr nur mit dem Aufdecken und der Propaganda der Notwendigkeit sozialistischer Umgestaltung begnügen kann, sondern Position zu den konkreten Formen stáatsmonopolistischer Vergesellschaftung beziehen mu\}. Dies ist heute ein wesentlicher Inhalt demokratischer und antimonopolistischer Alternativen.

\section{Die Kräfte der Umgestaltung und die Kampflinien}

Die Frage nach dem Subjekt progressiver gesellschaftlicher Veränderungen ist im Kapitalismus die Frage nach der Arbeiterklasse und den mit ihr verbündeten Kräften und ihrer Rolle in den ökonomischen, sozialen, politischen und ideologischen Kämpfen.

Freilich ist die Arbeiterklasse in jeder Entwicklungsphase der Veränderung ihrer inneren Struktur, ihrer konkreten Existenzgrundlagen und ihres sozialen Profils unterworfen, und das wird in verstärktem Maßje für eine Periode der Entwicklungsbeschleunigung und des Umbruchs gelten, wie sie vor uns liegt. Diese Fragen sind in jüngerer Zeit hinreichend erörtert und untersucht worden. ${ }^{7}$ Entscheidend ist, ob infolge sich differenzierender Arbeits- und Lebensbedingungen die einzelnen Schichten stärker auseinanderdriften oder ob sich die Tendenzen des Zusammenhangs, eines einheitlichen Klasseninteresses, das objektiv immer gegeben ist, im sozialen und politischen Prozeb geltend machen. Im Zentrum steht dabei auch die Frage, welche Schichten und Gruppen die Klasseninteressen derart zu artikulieren vermögen, daf sie zum Kristallisations- und Orientierungspunkt der gesamten Klasse werden können. Es geht dabei nicht schlechthin um die Erkenntnis des sozialökonomischen und gewerkschaftlichen Kerns der Arbeiterklasse - dał dies heute und in der kommenden Periode die Arbeiterschaft und die um sie gruppierten Gruppen in den Grofbetrieben der materiellen Produktion sein werden, ist kaum zu bezweifeln. Aber die

7 Vgl. dazu die Beiträge in: IMSF-Jb 6 (1983). Schwerpunkt: Arbeiterklasse in den 80er Jahren; IMSF-Jb 7 (1984). Schwerpunkt: Arbeiterklasse im Betrieb 
noch offene Frage ist, welche Gruppen der Arbeiterklasse für die Zukunft jene Rolle in einem stärker durch nichtmanuelle Arbeit, Freizeit, Bildung, Reproduktionsfragen und demokratische Partizipation geprägten Milieu einnehmen werden, die in einer früheren Periode und bis in die Gegenwart die Facharbeiter mit städtischer Bindung und Arbeiterherkunft spielten. Noch offen ist auch, aus welchen Quellen der neue Produzententyp der Arbeiterklasse jenes kollektive Selbstbewuftsein gewinnen wird, das für die Konstituierung der Arbeiterklasse im Betrieb eine zentrale Rolle spielt.

Diese Quellen werden weniger die beruflich-fachliche Kompetenz und der Produzentenstolz sowie das Kollektivbewuģtsein der Arbeitsgruppe sein, wie es für Arbeiter der "alten" Fabrik charakteristisch war und ist, sondern kollektive Stärke wird sich mehr aus der Vertretung der sozialen Interessen und dem Bewufftsein, die personellen Träger komplexer Produktionszusammenhänge zu sein, ergeben und den daraus resultierenden Ansprüchen auf Kontrolle und Beteiligung an der Gestaltung der technologischen und organisatorischen Zusammenhänge. Die Zusammenhänge zwischen Produktion und Reproduktion, Arbeits- und Lebensweise werden sich in vieler Hinsicht neu gestalten und auch die Bedürfnisse, Orientierungen, Wertvorstellungen usw. beeinflussen. Die Produktion und gesellschaftliche Arbeit und somit auch der Betrieb werden die wesentliche Basis der Konstitution der Arbeiterklasse, ihrer gewerkschaftlichen Organisation, ihrer Vertretungskörperschaften bleiben, aber die übrigen Lebenssphären werden auch für die Klassenkonstituierung einen Bedeutungsgewinn verzeichnen. Die Wechselbeziehungen zwischen betrieblicher Klassenorganisation und auferbetrieblichem Lebensmilieu werden auf neue Art intensiver werden und sich nicht nur als Einbahnstrafe gestalten.

Unter solchen Bedingungen wird die Organisierung der kulturellen Interessen der verschiedenen Schichten der Arbeiterklasse und der Kampf um kulturelle und ideologische Hegemonie im gesamtgesellschaftlichen Mafstab für die Arbeiterbewegung zu einer zentralen Frage werden; damit treten die Bündnisbeziehungen mit der Intelligenz und die Entwicklung in der Intelligenz als erstrangige Frage auf die Tagesordnung. ${ }^{8}$ Annäherungsprozesse in der sozialen Lage, die Ausprägung des Lohnarbeitscharakters dieser Schicht, eine stärkere Verschiebung der Rekrutierung in die Arbeiterklasse u. a. werden einen solchen Prozef begünstigen. Dies zeigen heute schon Entwicklungen in der jüngeren Generation. Diese Entwicklungen der Arbeiterklasse an "sich" und ihrer Stellung in der Gesellschaft stellen ihre gewerkschaftlichen und politischen Formationen vor neue Aufgaben, die schon heute deutlich sichtbar sind. Sie sind aus der Analyse der jüngsten Kämpfe und Bewegungen klar erkennbar.

Diese Kämpfe sind in hohem Małe durch die Krisensituation und die Einführung der neuen Technik geprägt. Als ersten Ty $p$ von Kämpfen könnte man jenen betrachten, wo die gewerkschaftliche Organisation unter Mobilisierung des gewerkschaftlich organisierten Kerns der Arbeiterklasse in Aktion tritt. Selbst gegen die geschlossene Front des SMK konnten die Handlungsfähigkeit unter Beweis gestellt und Teilerfolge erzielt werden. Von Bedeutung war vor allem auch, da\} Fragen der Kontrolle der Technik und die Arbeitszeitverkürzung zu Mobilisierungshebeln werden konnten. Gleichwohl zeigten sich auch die Probleme und die derzeitigen Grenzen für die Arbeiterbewegung (Haltung und gewerkschaftliche Organisierung der Angestellten einschlieflich der betrieblichen Intelligenz -, übergreifende gewerkschaftliche Solidarität und Überleitung auf die Ebene des Klassenkampfes im nationalen Rahmen, Kampfformen und Streikfähigkeit u. a.).

Auch beim zweiten Typ von Kämpfen, den Bewegungen für Erhaltung von Arbeitsplätzen, Betrieben, Standorten usw., zeigten sich diese Probleme. Es waren in der Regel im betrieblichen und regionalen Raum breite soziale und politische Bündnisse möglich. Die Erfolge waren jedoch begrenzt. In den Mittelpunkt trat die Frage alter-

8 Vgl. Intelligenz, Intellektuelle und Arbeiterbewegung in Westeuropa. IMSF-Arbeitsmaterialien 16. Frankfurt a. M. 1985; H. Lange: Die lohnabhängigen Naturwissenschaftler und Ingenieure. In : IMSF-Jb 7 (1984). S. 233 
nativer wirtschaftspolitischer Konzeptionen. Ihre Bedeutung dürfte sich in Zukunft noch erhöhen.

Bewegungen gegen den Sozial- und Demokratieabbau, die an die Adresse der Regierung gerichtet sind, könnten als dritten Kampttyp gesehen werden. Sie haben von vornherein betriebs- und branchenübergreifenden Charakter, beziehen sich auf die Reproduktionsinteressen und Kampfmöglichkeiten der Gewerkschaften, entfalten sich meist im nationalen Rahmen und bedienen sich der Kampfformen der auferparlamentarischen Bewegung. Auch hier bestand das Hauptproblem in der Herbeiführung einer breiten aktiven Massenresonanz. Ähnliche Probleme zeigen sich auch bei der Entwicklung der Arbeitslosenbewegung. Offensichtlich sind die Erfolgsaussichten stark an die Verknüpfung sozialer und demokratischer Aspekte gebunden. Dies wird in Zukunft noch mehr gelten.

Für die Handlungsfähigkeit der Gewerkschaften und der Betriebsbelegschaften haben sich der Bruch mit der Ideologie und Praxis der Sozialpartnerschaft und die Stärkung der klassenautonomen Strömung in der Arbeiterklasse und ihren gewerkschaftlichen Organisationen als wichtigstes Problem herausgestellt. Obwohl mit fortschreitender Krise die bestehenden sozialpartnerschaftlichen Muster auch unter den Druck des Kapitals und der konservativen Kräfte der Regierung gerieten, bestehen keine Gründe für die Annahme, daf̧ die westdeutsche Bourgeoisie auf die Linie der Integration und Sozialpartnerschaft verzichten könnte oder würde. Im Rahmen einer reformistisch-etatistischen Entwicklungsvariante des SMK müfte vielmehr mit einer Verstärkung dieser Tendenz gerechnet werden. Für das Konzept klassenautonomer Gewerkschaftspolitik wird es entscheidend sein, ob frühzeitig die Probleme und Konflikte der Umbruchperiode thematisiert und mit einer Strategie gesamtgesellschaftlicher Demokratisierung antimonopolistischen Charakters verbunden werden können.

Im übertragenen und auf die politische Ebene transformierten Sinne wird diese Auseinandersetzung auch die Sozialdemokratie prägen. Es ist gegenwärtig noch nicht auszumachen, ob Impulse aus dem gewerkschaftlichen und Arbeiterbereich eine stärkere Linkswendung der reformistischen Strömung befördern können oder ob sich die linken Hauptkräfte unter den Fahnen eines modifizierten Ökoreformismus sammeln werden. Für eine progressive Entwicklung der Sozialdemokratie werden schlieflich wesentliche Bedingungen die Stabilisierung und Autonomie der auferparlamentarischen Massenbewegungen, die Fortexistenz einer radikaldemokratischen politischen Formation auf parlamentarischer Ebene und die Stärkung der revolutionären marxistischen Strömung der Arbeiterbewegung, ihrer Partei und Organisationen, sein.

Was den revolutionären marxistischen Flügel der Arbeiterbewegung betrifft, der gegenwärtig in der BRD nicht über Masseneinfluf verfügt, so kommt ihm unter allen denkbaren Konstellationen die Rolle des vorwärtstreibenden Elementes zu. Ihm werden aber in der kommenden Periode sowohl aus der Veränderung der internationalen Situation als auch aus den Konflikten und Kämpfen des Umbruchs neue Kräfte zuwachsen. $\mathrm{Ob}$ er politische und ideologische Hegemoniefähigkeit zu entwickeln vermag, wird nicht nur von seiner konsequenten und kontinuierlichen Orientierung auf die unmittelbaren Probleme der Arbeiterklasse abhängen, sondern auch von seinem Vermögen, sich für die fortgeschrittensten Kräfte aus den sozialen und demokratischen Bewegungen, aus der Intelligenz, der Jugend, der Frauenbewegung, zu öffnen und für sie Attraktivität zu entwickeln. ${ }^{9}$

Mit der Partei der Grünen und ihren parlamentarischen Erfolgen ist eine politische Formation entstanden, die Bewegung in die politische Szene der BRD gebracht hat. Sie ist in ihrer Haupttendenz politischer Ausdruck einer radikal-demokratischen Strömung, die ihre wichtigste Basis in den jüngeren Generationen der Intelligenz und der lohnabhängigen Mittelschichten hat, die mit besonderer Sensibilität auf die De-

9 Vgl. Thesen und Materialien des 8. Parteitages der DKP (2.-4. Mai 1986 in Hamburg). In: Unsere Zeit (Düsseldorf). 3., 6., 7., 10. Mai 1986 
formationsprozesse des SMK reagieren. Die Stabilität und das Wachstum dieser Gruppierung liegt im Interesse einer fortschrittlichen Entwicklung der BRD. Unabhängig von ihrer Entwicklung als Parlamentspartei spricht alles dafür, daf̧ sich in der kommenden Periode die soziale und politische Basis einer radikal-demokratischen Grundströmung erweitern wird.

Die vom Standpunkt der Entwicklung der Kräfte gesellschaftlicher und politischer Veränderungen wichtigste neue Erscheinung ist seit Mitte der 70er Jahre die Entstehung und Wirksamkeit demokratischer und sozialer Massenbewegungen, die vielfach unter den Begriff "neue soziale Bewegungen“ geschlagen werden. ${ }^{10}$ Wie die Analyse zeigt, ist dieser Begriff nur für einen Sektor des Gesamtspektrums korrekt. Das Gesamtspektrum reicht von der Friedensbewegung über die Ökologiebewegung, die Bürgerinitiativen mit den verschiedensten Anliegen, die Frauenbewegung, die Minderheitenbewegung, die Bewegung gegen die Berufsverbote, Bewegungen gegen die Sozialdemontage, die Bewegung gegen die Volksaushorchung usw. bis zur antifaschistischen Bewegung, $z u$ den antiimperialistischen Solidaritätsbewegungen usw. Diese Bewegungen haben das politische Klima in der BRD nachhaltig verändert und den Boden für die Verstärkung linker Orientierungen bereitet. Sie haben neue Kräfte für den Kampf gegen die Ausdrucksformen des sozialen Antagonismus unter den Bedingungen des SMK in Bewegung gebracht, ohne von dessen Grundstrukturen aufzubrechen und sich ideologisch daran zu orientieren. In ihnen haben Kräfte aus der Intelligenz eine vielfach dominierende und ideologisch hegemoniale Rolle spielen können. Es ist von Bedeutung, dał sich das Protestpotential auferhalb des etablierten Parteienspektrums formierte, jeweils auf wechselnde Konfliktfelder konzentrierte, und ein Teil dieser Bewegungen als sogenannte Einpunktbewegungen einen Zyklus durchlief und nicht von dauerhaftem Bestand war. Vom Abflauen dieser oder jener Bewegung kann aber nicht auf ein Schrumpfen des Protestpotentials geschlossen werden.

Versucht man ihre Rolle in der kommenden Periode zu beurteilen, dann muf zuerst davon ausgegangen werden, dał sich die Widersprüche des SMK auf vielen Gebieten verschärfen und an immer neuen Punkten Konflikte aufbrechen werden. Das spricht dafür, dał diese Bewegungen ein an Bedeuting wachsendes Element des politischen Lebens und der fortschrittlichen Bewegung überhaupt werden. Ob und inwieweit Orientierungen der Arbeiterbewegung größeres Gewicht in diesen Bewegungen erlangen können, hängt in erster Linie von der Dynamik der Arbeiterklasse und ihrer Bewegung ab. Es liegt aber auf der Hand, daf sich aus dem Zusammenwirken beider Kräfte in der Aktion jenes Potential entbinden und formieren kann, das grundlegende gesellschaftliche Änderungen durchzusetzen in der Lage sein wird.

Wenn wir von der Gegenwart jene knapp 15 Jahre zu überblicken versuchen, die uns vom Jahr 2000 noch trennen, dann scheinen die Hauptthemen der sozialen und politischen Auseinandersetzungen in den Konturen erkennbar: Sicherung des Friedens, Abrüstung, Arbeitslosigkeit, Arbeitszeitverkürzung, Umweltprobleme, Gestaltung der Arbeit, soziale Kontrolle von Technik und Wissenschaft, Demokratisierung, Hilfe für die "dritte Welt", antiimperialistische Solidarität.

Wenn diese Zukunft als Zukunft des Klassenkampfes erschlossen werden soll, dann darf niemals übersehen werden, daf auch die herrschende Klasse als handelndes und die Gesellschaft beherrschendes Subjekt auf der Bühne sein wird. Man muf beachten, daf mit zunehmenden Krisen- und Zersetzungserscheinungen sich destruktiv-reaktionäre Kräfte formieren werden, die in Verbindung mit monopolkapitalistischen Interessen schnell gesellschaftliches Gewicht erlangen können und auf die Veränderung nach rechts drängen werden. Der Weg in eine demokratische Zukunft kann nur offen gehalten werden, wenn eine solche Möglichkeit verhindert wird.

10 Vgl. zu diesem Komplex: IMSF-Jb 5 (1982) mit dem Schwerpunkt "Neue soziale Bewegungen und Arbeiterbewegung".

(Verf.: Dr. sc. oec.; Institut für Marxistische Studien und Forschungen, Oberlindau 15, Frankfurt a. M., D - 6000) 